\title{
Green epithelium revealed by narrow-band imaging (NBI): a feature for practical assessment of extent of gastric cancer after $H$. pylori eradication
}

\section{(๑) $\circledast \ominus$}

\author{
Authors \\ Kazuyoshi Yagi ${ }^{1}$, Itsuo Nagayama ${ }^{1}$, Takahiro Hoshi ${ }^{1}$, Satoshi Abe' ${ }^{1}$, Shin-ichi Morita ${ }^{1}$, Takeshi Suda ${ }^{1}$, Go Hasegawa ${ }^{2}$, \\ Yu-ichi Sato ${ }^{3}$, Shuji Terai ${ }^{4}$
}

Institutions

1 Department of Gastroenterology and Hepatology, Uonuma Institute of Community Medicine, Niigata University Medical and Dental Hospital, Niigata, Japan

2 Department of Pathology, Uonuma Institute of Community Medicine, Niigata University Medical and Dental Hospital, Niigata, Japan

3 Department of Internal Medicine, Niigata Prefectural Yoshida Hospital, Niigata, Japan

4 Division of Gastroenterology and Hepatology, Niigata University Graduate School of Medical and Dental Sciences, Niigata, Japan

submitted 21.3.2018

accepted after revision 9.8.2018

Bibliography

DOI https://doi.org/10.1055/a-0746-3449 |

Endoscopy International Open 2018; 06: E1289-E1295

(c) Georg Thieme Verlag KG Stuttgart · New York

ISSN 2364-3722

Corresponding author

Kazuyoshi Yagi, MD, Department of Gastroenterology and Hepatology, Uonuma Institute of Community Medicine, Niigata University Medical and Dental Hospital, 4132 Urasa, Minami-Uonuma, Niigata 949-7302, Japan

Fax: +81-25-777-5067

yagikazu@pop12.odn.ne.jp

\section{ABSTRACT}

Background and study aims Assessment of the extent of gastric cancer detected by endoscopy after successful eradication of $H$. pylori is often difficult even with narrowband imaging (NBI)-magnifying endoscopy. Using the latter modality, it has often been noted that the cancerous area has a brownish color surrounded by green epithelium (GE). If the histological origin of this GE could be clarified, it could be of practical use for endoscopic assessment of the extent of cancer.

Materials and methods The endoscopic appearance of gastric cancer after eradication therapy was classified into four types: (A) cancer brownish, surrounding mucosa green; (B) cancer brownish, surrounding mucosa brownish; (C) cancer green, surrounding mucosa brownish; and (D) cancer green, surrounding mucosa green. A histological series of sections taken from tissues in each endoscopic view was stained with HE, and also for CDX2, MUC2, CD10 and MUC5AC. Staining intensity was evaluated by microscopy using a visual analog scale ranging from 0 to $3+$ : intensities of 0 and $1+$ were rated as negative, and those of $2+$ and $3+$ as positive. Positive or negative immunostaining was examined for each type of endoscopic appearance A-D.

Results Among 42 lesions examined, 16 were type A, 18 type B, 6 type $C$ and 2 type D. MUC2 was positive in the surrounding mucosa in $100 \%$ of type $A$ and $D$ cases, but in only $28 \%$ and $17 \%$ of type B and C cases, respectively.

Conclusions GE observed by NBI-endoscopy corresponds to MUC2-positive mucosa and is thought to be a feature for practical assessment of the extent of cancer.

\section{Introduction}

We have reported that gastric cancer detected after successful eradication of Helicobacter pylori has a gastritis-like appearance by endoscopy, often making diagnosis difficult [1]. As assessment of the extent of cancer is essential for successful endoscopic submucosal dissection (ESD), these gastritis-like cancers pose a diagnostic problem for endoscopists. Assessment of the extent of cancer has hitherto been based on irregularities of mucosal structure and micro-vessels revealed by narrow-band imaging (NBI) magnifying endoscopy [2,3]. However, gastric cancer after $H$. pylori eradication therapy often shows a mucosal structure similar to that of surrounding areas of gastritis [1].

In our studies of gastric cancer after eradication therapy using NBI endoscopy [1], we have noticed that a proportion of such cases have a brownish-colored cancerous area and a green-colored surrounding mucosa (gastritis mucosa). If sufficiently accurate, this difference in color could be applicable for 

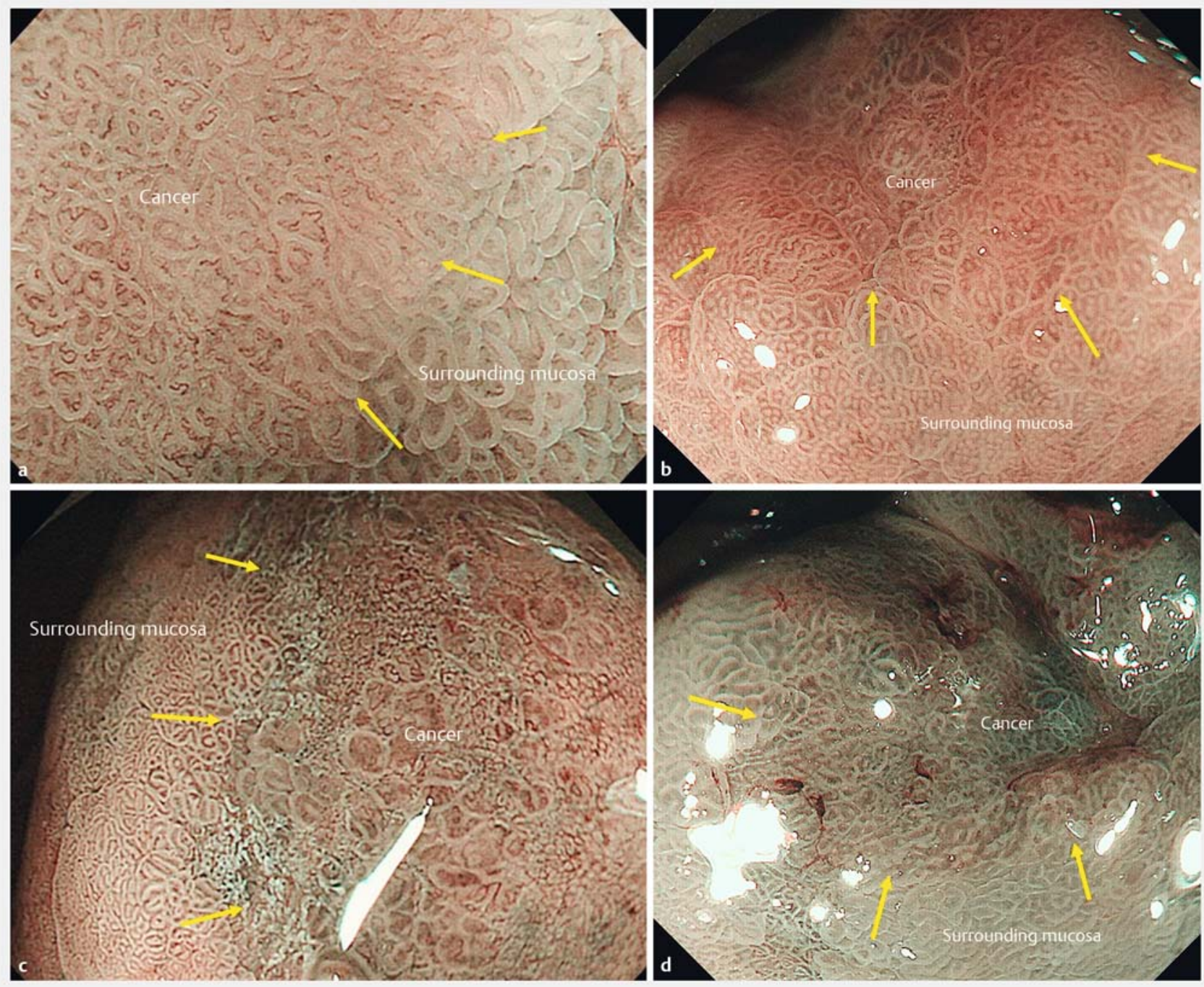

- Fig. 1 a NBI-endoscopic appearance of brownish cancerous area and green surrounding mucosa. b NBI-endoscopic appearance of brownish cancerous area and brownish surrounding mucosa. c NBI-endoscopic appearance of green cancerous area and brownish surrounding mucosa. d NBI-endoscopic appearance of green cancerous area and green surrounding mucosa.

determining the borderline between the cancerous area and the surrounding mucosa.

However, the histological origin of the brown- or green-colored mucosa has not been investigated. For more than 19 years we have been comparing the findings of endoscopy and magnifying endoscopy with standard histological findings, including gastric cancer after eradication therapy [1]. If the histological origin of the green epithelium, demonstrated by NBIendoscopy could be clarified, it might become applicable as a reliable marker for indicating the extent of the cancerous area. Therefore, using NBI-endoscopy, we performed the present study to clarify the histological origin of the green epithelium.

\section{Materials and methods}

Study design and setting

From among gastric cancers treated by ESD at Niigata Prefectural Yoshida Hospital between 2004 and 2017, 44 that had been diagnosed after $\mathrm{H}$. pylori eradication therapy, for which findings of imaging including NBI magnifying endoscopy corresponded to the histological findings, were included in this study.

We defined gastric cancer after successful $H$. pylori eradication as that which had been detected and diagnosed at least 1 year after the therapy. The efficacy of $H$. pylori eradication treatment was evaluated by the ${ }^{13} \mathrm{C}$-urea breath test (UBIT, Otsuka, Tokushima, Japan) and the $\mathrm{H}$. pylori stool antigen test (Premier Platinum HpSA; Meridian, Cincinnati, Ohio, United States). If the results were negative in both tests, we judged that $\mathrm{H}$. pylori eradication had been successful. These 44 gastric cancers were observed by NBI-magnifying endoscopy and the areas sur- 
rounding the cancer were marked by high-frequency electrocautery before ESD to facilitate radical resection and assessment of the histological features corresponding to the findings of magnifying endoscopy.

Before ESD, many photographs were taken of the border between the cancerous area and the surrounding mucosa. From these $\mathrm{NBI}$ endoscopy photographs, areas in which green or brownish coloration were recognizable were chosen. We also confirmed that histological specimens corresponding to these endoscopically visualized areas were available, and that the tissue had not been injured sufficiently to rule out histological assessment. Areas of gastric cancer matching these criteria were chosen for the current study.

\section{Endoscopic evaluation}

Endoscopic examinations were performed using a magnifying endoscope (GIF-H260Z; Olympus Medical Systems, Tokyo, Japan) and an electronic endoscopic system (EVIS LUCERA Spectrum; Olympus Medical Systems).

The endoscopic appearance of the chosen areas was classified into four types: (A) cancer brownish, surrounding mucosa green ( $>$ Fig.1a); (B) cancer brownish, surrounding mucosa brownish ( Fig. 1b); (C) cancer green, surrounding mucosa brownish ( $\triangleright$ Fig. 1c); and (D) cancer green, surrounding mucosa green ( $\triangleright$ Fig. 1d). Classification was performed by two of the authors, KY and YS, who were expert endoscopists. If their opinions differed, the case was discussed and a final consensus decision was reached. For any area in which a mixture of green and brownish was observed, the predominant color was chosen.

\section{Histological evaluation}

A series of histological sections of areas in each field evaluated by endoscopy was prepared and stained with HE (hematoxylineosin), and also using immunohistochemistry for caudal-related homeobox protein 2 (CDX2), mucin core protein 2 (MUC2), cluster of differentiation 10 (CD10), and mucin core protein $5 A C(M U C 5 A C)$.

CDX2 is an intestine-specific transcription factor reported to be important for development and maintenance of the intestinal epithelium [4]. MUC2 is a core protein in goblet cells, and CD10 indicates the brush border of absorptive cells [5]. MUC5AC is a core protein present in surface mucous cells [5].

The specimens chosen for this study were each cut serially into five thin sections, which were stained with $\mathrm{HE}$, and also immunohistochemically for CDX2 (clone DAK-CDX2; Ready-toUse: Dako), MUC2 (clone CCP58; Ready-to-Use: Dako), CD10 (clone 56C6, 1:100; Novocastra) and MUC5AC (clone CLH2; Ready-to-Use: Dako).

One author $(\mathrm{GH})$ evaluated the staining intensity of CDX2, MUC2, CD10 and MUC5AC by microscopy using a visual analog scale ranging from 0 to $3+$. The intensity was defined as: 0 , gland or cell. $1+$, staining in less than one third of all glands or cells; $2+$, staining in one third to two thirds of all glands or cells; or $3+$, staining in over two thirds of all glands or cells. Intensities of 0 and $1+$ were assessed as negative, and 2+ and 3+ as positive. Positivity or negativity of immunostaining with each antibody was examined in each endoscopically classified type of histology. In addition, all combinations of MUC2, CD10 and MUC5AC, which are proteins present in the cytoplasm and brush border, were clarified and statistically evaluated.

\section{Statistical analysis}

The positivity and negativity of immunostaining with each antibody in the brownish and green areas of cancerous tissue and surrounding mucosa were compared statistically using Fisher's exact test. Statistical analyses were performed using JMP software, version 13.2 (SAS Institute Inc., Cary, North Carolina, United States). For comparisons between multiple groups, after Fisher's exact test, we employed the Bonferroni correction to set the significance level to $0.05 / a$ where "a" represents the number of tests.

\section{Results}

Of 44 lesions, 42 met the above-mentioned criteria. All of the lesions were differentiated adenocarcinomas and intramucosal cancers.

The time between eradication therapy and detection of cancer ranged from 1 year to 15 years (mean: 7 years).

\section{Endoscopic evaluation}

Sixteen cases were evaluated endoscopically as type A, 18 as type B, 6 as type $C$, and 2 as type $D$. There was no relationship between the endoscopically assessed color of the surrounding mucosa, i.e. brownish or green, and the period elapsed after H. pylori eradication.

\section{Histological evaluation}

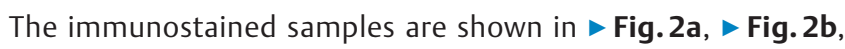
- Fig. 2c and $>$ Fig.2d. P Fig.2a represents histological findings corresponding to the endoscopic view shown in $\mathbf{F i g . 1} \mathbf{1 a}$. - Fig. $\mathbf{2 b}$ represents histological findings corresponding to the

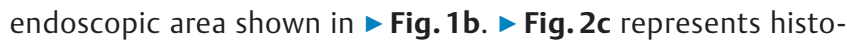
logical findings corresponding to the endoscopic area shown in - Fig. 1c. P Fig. 2d represents histological findings corresponding to the endoscopic area shown in $\mathbf{F i g . 1 d}$.

Each histological finding corresponding to each endoscopic finding was evaluated as shown in > Fig. 1a, > Fig. 1b, > Fig. 2a, and $\triangleright$ Fig. 2 b.

Immunohistochemical positivity or negativity in each endoscopic type is shown in $>$ Table 1 . The surrounding mucosa in types A and D, which appeared green, was MUC2-positive in $100 \%$ of cases, of which $56 \%$ and $50 \%$ were positive for CD 10 , respectively. On the other hand, the surrounding area in types $B$ and C, which appeared brownish, was MUC2-positive in $28 \%$ and $17 \%$ of cases, respectively. The cancerous area in types $C$ and D, which appeared green, was MUC2-positive in $67 \%$ and $100 \%$ of cases, respectively, the frequency being higher than in types A and B, i. e. $19 \%$ and $17 \%$, respectively.

MUC2 was confirmed to have a statistically significant relationship with the green area in cancerous tissue ( $\nabla$ Table $2, P=$ 0.0036). In the surrounding mucosa, expression of CDX2, MUC2 and CD10 was confirmed to have a significant relationship to the green area ( $\vee$ Table $2, P<0.0001, P<0.0001$ and $P=$ 


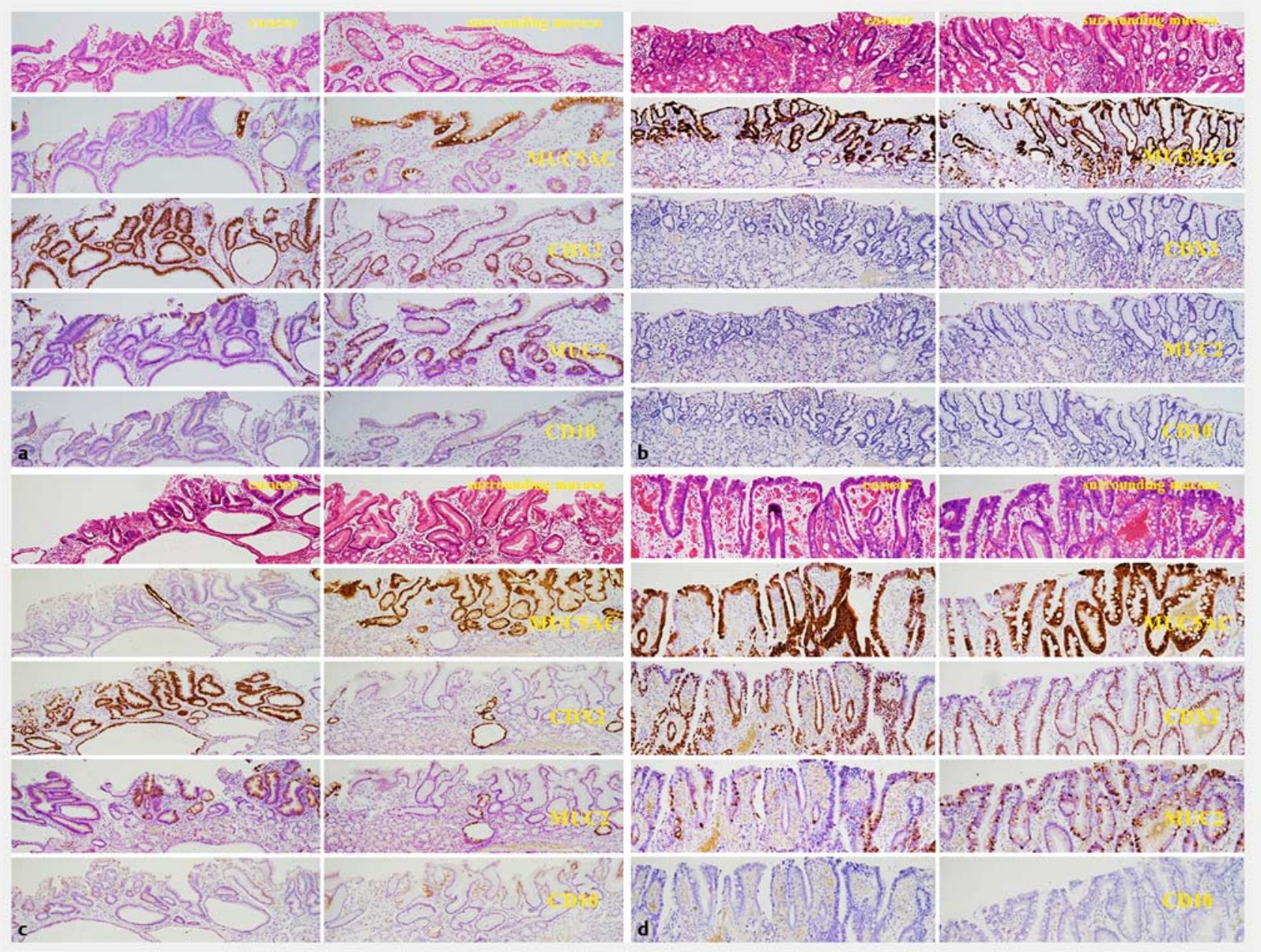

- Fig. 2 a Histological features of $>$ Fig. 1 a. The left side is the cancerous area and the right side is the surrounding mucosa. The cancerous area has the following scores: MUC5AC 1+, CDX2 3+, MUC2 1+, CD10 1+. The surrounding mucosa has the following scores: MUC5AC 3+, CDX2 3+, MUC2 $3+$, CD10 1+. b Histological features of $\mathbf{F i g . ~} \mathbf{1 b}$. The left side is the cancerous area and the right side the surrounding mucosa. The cancerous area has the following scores: MUC5AC 3+, CDX2 3+, MUC2 1+, CD10 0+. The surrounding mucosa has the following scores: MUC5AC $3+$, CDX2 $0+$, MUC2 $0+, C D 100+$. c Histological features of $>$ Fig. 1c. The left side is the cancerous area and the right side the surrounding mucosa. The cancerous area has the following scores: MUC5AC 1+, CDX2 3+, MUC2 2+, CD10 1+. The surrounding mucosa has the following scores: MUC5AC $3+$, CDX2 1+, MUC2 1+, CD10 0+. d Histological features of $\mathbf{p i g}$. 1d. The left side is the cancerous area and the right side the surrounding mucosa. The cancerous area has the following scores: MUC5AC 3+, CDX2 3+, MUC2 2+, CD10 0+. The surrounding mucosa has the following scores; MUC5AC 3+, CDX2 3+, MUC2 3+, CD10 0+.

0.0014 , respectively). Expression of MUC5AC was confirmed to have a significant relationship to the brownish area in the surrounding mucosa ( $>$ Table $2, P=0.0104)$. The combined results for expression of MUC2, CD10 and MUC5AC are shown in > Table 3. In the cancerous area, "MUC2 only" was significant correlated with the green area ( $\triangleright$ Table $3, P=0.0079$ ). In the surrounding mucosa, "MUC5AC only" was significantly correlated with the brownish area ( $\triangleright$ Table 3, $P=0.0001$ ).

\section{Discussion}

It has been reported that gastric cancer detected after successful eradication of $H$. pylori tends to show gastritis-like features $[1,6]$. Such a gastritis-like mucosal pattern is often observed by NBI magnifying endoscopy, making it difficult to assess the extent of gastric cancer [1,6]. Using NBI-endoscopy, we have often noticed that the cancerous area appears brownish and the surrounding mucosa green. Our impression has been that the green epithelium observed by NBI endoscopy might be intestinal metaplasia, although there were no histological data to support this possibility. The light blue crest (LBC) is known to be a feature of intestinal metaplasia observed by NBI magnifying endoscopy [7]. LBC appears as a fine, blue-white line on the crests of the epithelial surfaces and gyri, and is reported to correspond to the brush border of the absorptive epithelium, denoting the presence of CD10 [7]. Initially, we speculated that the green epithelium may have been due to the influence of the LBC. However, gastric and intestinal (GI) mixed-type intes- 
Table 1 Number and ratio of cells positive for CDX2, MUC2, CD10 and MUC5AC in the cancerous area and surrounding mucosa for each of the endoscopically assessed color types.

\begin{tabular}{|c|c|c|c|c|c|c|c|c|}
\hline & \multicolumn{4}{|c|}{ Cancerous area } & \multicolumn{4}{|c|}{ Surrounding mucosa } \\
\hline & CDX2 & MUC2 & CD10 & MUC5AC & CDX2 & MUC2 & CD10 & UMUC5AC \\
\hline Type A $(n=16)$ & $16(100 \%)$ & $3(19 \%)$ & $3(19 \%)$ & $6(38 \%)$ & $16(100 \%)$ & $16(100 \%)$ & $9(56 \%)$ & $9(50 \%)$ \\
\hline Type B $(n=18)$ & $16(89 \%)$ & $3(17 \%)$ & $5(28 \%)$ & $6(34 \%)$ & $5(28 \%)$ & $5(28 \%)$ & $2(11 \%)$ & $17(94 \%)$ \\
\hline Type $C(n=6)$ & $6(100 \%)$ & $4(67 \%)$ & $0(0 \%)$ & $1(17 \%)$ & $1(17 \%)$ & $1(17 \%)$ & $0(0 \%)$ & $5(83 \%)$ \\
\hline Type D ( $n=2)$ & $2(100 \%)$ & $2(100 \%)$ & $0(0 \%)$ & $2(100 \%)$ & $2(100 \%)$ & $2(100 \%)$ & $1(50 \%)$ & $1(50 \%)$ \\
\hline
\end{tabular}

- Table 2 Number and ratio of cells positive for CDX2, MUC2, CD10 and MUC5AC in brownish and green areas of cancerous tissue and surrounding mucosa.

\section{Cancerous area}

\begin{tabular}{|l|l|l|l|l|}
\hline & CDX2 & MUC2 & CD10 & MUC5AC \\
\hline Brownish cancerous area $(n=34)$ & $32(94.1 \%)$ & $6(17.7 \%)$ & $8(23.5 \%)$ & $12(35.3 \%)$ \\
\hline Green cancerous area $(n=8)$ & $8(100 \%)$ & $6(75.0 \%)$ & $0(0 \%)$ & $3(37.5 \%)$ \\
\hline$P$ value & 1.0000 & 0.0036 & 0.3163 & 1.0000 \\
\hline
\end{tabular}

We used the Bonferroni correction to set the significance level to $0.05 / 4=0.0125$

Surrounding mucosa

\begin{tabular}{|l|c|c|c|c|}
\hline & CDX2 & MUC2 & CD10 & MUC5AC \\
\hline Brownish surrounding mucosa $(n=24)$ & $6(25.0 \%)$ & $6(25.0 \%)$ & $2(8.3 \%)$ & $22(91.7 \%)$ \\
\hline Green surrounding mucosa $(n=18)$ & $18(100 \%)$ & $18(100 \%)$ & $10(55.6 \%)$ & $10(55.6 \%)$ \\
\hline$P$ value & $<0.0001$ & $<0.0001$ & 0.0014 & 0.0104 \\
\hline
\end{tabular}

We used the Bonferroni correction to set the significance level to $0.05 / 4=0.0125$

- Table 3 Number and ratio of cells positive for various combination of MUC2, CD10 and MUC5AC in brownish and green areas of cancerous tissue and surrounding mucosa.

Cancerous area

\begin{tabular}{|c|c|c|c|c|c|c|}
\hline & $\begin{array}{l}\text { (a) MUC2 + } \\
\text { CD10 \# }\end{array}$ & $\begin{array}{l}\text { (b) MUC2 + } \\
\text { MUC5AC }\end{array}$ & $\begin{array}{l}\text { (c) MUC2 } \\
\text { only }\end{array}$ & $\begin{array}{l}\text { (d) CD10 } \\
\text { only }\end{array}$ & $\begin{array}{l}\text { (e) MUC5AC } \\
\text { only }\end{array}$ & $\begin{array}{l}\text { (f) non- } \\
\text { staining }\end{array}$ \\
\hline Brownish cancerous area $(n=34)$ & $4(11.8 \%)$ & $0(0 \%)$ & $2(5.9 \%)$ & $4(11.8 \%)$ & $12(35.3 \%)$ & $12(35.3 \%)$ \\
\hline Green cancerous area $(n=8)$ & $0(0 \%)$ & $2(25.0 \%)$ & $4(50.0 \%)$ & $0(0 \%)$ & $1(12.5 \%)$ & $1(12.5 \%)$ \\
\hline$P$ value & 0.5723 & 0.0325 & 0.0079 & 0.5723 & 0.3983 & 0.3983 \\
\hline \multicolumn{7}{|l|}{ Surrounding mucosa } \\
\hline & $\begin{array}{l}\text { (a) MUC2 + } \\
\text { CD10+ MUC5AC }\end{array}$ & $\begin{array}{l}\text { (b) MUC2 + } \\
\text { CD10 }\end{array}$ & $\begin{array}{l}\text { (c) MUC2 + } \\
\text { MUC5AC }\end{array}$ & $\begin{array}{l}\text { (d) MUC2 } \\
\text { only }\end{array}$ & \multicolumn{2}{|c|}{ (e) MUC5AC only } \\
\hline Brownish surrounding mucosa $(n=24)$ & $1(4.2 \%)$ & $1(4.2 \%)$ & $3(12.5 \%)$ & $1(4.2 \%)$ & \multicolumn{2}{|l|}{$18(75 \%)$} \\
\hline Green surrounding mucosa $(n=18)$ & $3(16.7 \%)$ & $7(38.9 \%)$ & $7(38.9 \%)$ & $1(5.6 \%)$ & \multicolumn{2}{|l|}{$0(0 \%)$} \\
\hline$P$ value & 0.2972 & 0.0131 & 0.0701 & 1.0000 & \multicolumn{2}{|l|}{0.0001} \\
\hline \multicolumn{7}{|c|}{ We used the Bonferroni correction to set the significance level to $0.05 / 5=0.01$} \\
\hline \multicolumn{7}{|c|}{ We used the Bonferroni correction to set the significance level to $0.05 / 6=0.0083$} \\
\hline \multicolumn{7}{|c|}{$\begin{array}{l}\text { MUC2 + CD10 means that one gland was positive for both MUC2 and CD10, indicating a small-intestinal phenotype. } \\
\text { MUC2 + MUC5AC means that one gland was positive for both MUC2 and MUC5AC, indicating a gastric and intestinal mixed phenotype, or "incomplete intestinal } \\
\text { type" [5] } \\
\text { MUC2 + CD10 + MUC5AC indicates mosaic-like mucosa comprising a mixture of MUC2 + CD10 and MUC5AC-positive areas, making it difficult to divide into discrete }\end{array}$} \\
\hline
\end{tabular}


tinal metaplasia (IM) [5], which is MUC5AC-positive, MUC2-positive and CD10-negative [5], appears as green epithelium on $\mathrm{NBI}$ endoscopy. MUC5AC is a core protein of surface mucous cells and can be used as a gastric marker, whereas MUC2 is a core protein of goblet cells and an intestinal marker [5]. This suggested that the appearance of the green epithelium might be due to MUC2 (i.e., goblet cells), being unrelated to MUC5AC, thus prompting us to perform the current study. Here we found that the green epithelium demonstrated by NBI endoscopy corresponded to intestinal metaplasia containing abundant MUC2-positive (goblet) cells, as expected. Interestingly, cancerous areas containing MUC2-positive cells were also demonstrated as green epithelium by $\mathrm{NBI}$ endoscopy. However, the green epithelium in cancerous areas was less prominent than that of intestinal metaplasia in the surrounding mucosa, reflecting the fact that cancer has a smaller number of goblet cells.

The possibility that the green epithelial coloration demonstrated by NBI endoscopy is due to abundant MUC2-positive cells or goblet cells is supported by the fact that NBI light contains blue light as a component, which is observed as green on the screen if it is reflected by the mucosa, a greater degree of reflection creating a stronger green tone (personal communication from Olympus Co.). In this way, the blue light of NBI is thought to be reflected by MUC2-positive cells or goblet cells, appearing as green epithelium on the screen. The difference in the tone of green between cancerous areas and intestinal metaplasia is thought to depend on the number and degree of maturation of goblet cells.

Thus, the green epithelium in the surrounding mucosa and the green cancerous areas observed by NBI endoscopy obviously corresponded to presence of MUC2, especially the former.

In this study, we recognized a difference in CDX2 and MUC2 positivity between the cancerous area and the surrounding mucosa affected by gastritis. In cancerous areas, 40 cases were CDX-positive, among which 12 (30\%) were MUC2-positive, whereas in the surrounding mucosa, 24 cases were CDX2-positive, among which 24 (100\%) were MUC2-positive. Thus, it appeared that CDX2-positive cancerous cells tended to produce little or no MUC2. CDX2 is an intestine-specific transcription factor that is reportedly important for development and maintenance of the intestinal epithelium [4], being closely associated with intestinal phenotypic expression in human gastric carcinomas and intestinal metaplasia [8-15]. In addition, it has been reported that CDX2 has both tumor suppressor [10-15] and oncogenic activities [14], although it remains unclear whether it has an oncogenic role in gastric carcinogenesis. Furthermore, CDX2 is a more sensitive marker than MUC2 or CD10 for assessing presence of an intestinal phenotype from the very early stage of carcinogenesis [9]. Hayakawa et al. reported that CDX2 expression was evident in $89.1 \%$ of 313 cases of intestinal-type (by Lauren's classification [16]) early gastric adenocarcinoma [10]. In the current study, CDX2 expression was observed in $95 \%$ (40 of 42 ) of cases, thus supporting its relationship to oncogenic activity in the stomach.
Cancerous cells are thought to be so immature that they tend to produce little or no mucin. Therefore, if they are positive for the intestine-specific transcription factor CDX2, they might not produce a large amount of MUC2. On the other hand, intestinal metaplasia, which is a non-cancerous type of epithelium, comprises mature cells, and therefore, most of them produce MUC2 as a core protein of the intestinal phenotype if they are CDX2-positive. This may explain why NBI endoscopy demonstrates a difference in color between cancerous cells and the surrounding gastritis-affected mucosa due to the presence/paucity of MUC2.

The fact that intestinal metaplasia shows a green epithelium when viewed by NBI endoscopy would be very practical for assessing the extent of cancer before ESD. However, endoscopists need to be aware that a cancerous area rich in MUC2 may also show the green epithelium. Therefore, we emphasize that the green epithelium is due to presence of MUC2-positive goblet cells.

This green epithelium may also be useful for assessing the spread of intestinal metaplasia. The spread of intestinal metaplasia to the gastric body is reported to affect development of gastric cancer after $H$. pylori eradication therapy $[17,18]$. We have already reported a diagnostic system for OLGA- [19-20] and OLGIM- [21] based staging of gastritis using NBI-magnifying endoscopy [22]. However, it may be possible to recognize the spread of intestinal metaplasia through observation of the green epithelium by NBI endoscopy, thus allowing more accurate and practical staging of gastritis.

One doubt remains; although duodenal and colorectal mucosa are rich in goblet cells, these mucosae do not show a green epithelial coloration. We think that this may be due to a difference in the mucin of goblet cells between intestinal metaplasia and duodenal or colorectal mucosa, or a specific component of goblet cells in intestinal metaplasia. Although this issue will require further investigation, this phenomenon is considered useful for determining whether or not gastric mucosa contains intestinal metaplasia.

In addition, we noticed that intestinal metaplasia with CD10 positivity tended to show a stronger green tone. The brush border of intestinal metaplasia may be related to green epithelium. However, as no area of surrounding mucosa was both CD10-positive and MUC2-negative, the exclusive association of CD10 positivity with green epithelium could not be evaluated. We also attempted to compare the strength of the green tone between CD10-positive and CD10-negative MUC2-positive green epithelium, but the number of endoscopic pictures we obtained was insufficient for this purpose.

One limitation of this study was that the lesions included were observed to be cancerous before ESD, so that our endoscopic observations were focused on the cancerous areas and not on the surrounding mucosa. Accordingly, the endoscopic pictures of the surrounding mucosa were insufficient for detailed investigation. Furthermore, the number of lesions was small and detailed immunohistological investigations could not be performed. Therefore, based on our current results, we intend to design a more detailed study of the color of intestinal metaplasia using NBI endoscopy. 


\section{Conclusion}

In conclusion, the green epithelium observed by NBI endoscopy is thought to be related to presence of MUC2-positive cells or goblet cells, and also to CD10 or brush border, being a potentially practical feature for assessing the extent of the cancerous area. However, if the cancerous area includes MUC2-positive cells, it may also appear green. Knowledge of the histological origin of the green epithelium may facilitate endoscopic prediction of the presence and spread of intestinal metaplasia in the stomach.

\section{Acknowledgement}

We thank Yusuke Horiuchi, the Cancer Institute Hospital of Japanese Foundation for Cancer Research for statistical analysis.

\section{Competing interests}

None

\section{References}

[1] Saka A, Yagi K, Nimura S. Endoscopic and histological features of gastric cancers after successful Helicobacter pylori eradication therapy. Gastric Cancer 2016; 19: 524- 530

[2] Kaise M, Kato M, Urashima M et al. Magnifying endoscopy combined with narrow-band imaging for differential diagnosis of superficial depressed gastric lesions. Endoscopy 2009; 41: 310-315

[3] Yagi K, Nakamura A, Sekine A et al. Magnifying endoscopy with narrow band imaging for early differentiatedadenocarcinoma. Dig Endosc 2009; 20: 115-122

[4] Silberg DG, Swain GP, Suh ER et al. Cdx1 and cdx2 expression during intestinal development. Gastroenterology 2000; 119: 961 - 971

[5] Tsukamoto T, Mizoshita T, Tatematsu M. Gastric-and-intestinal mixed type intestinal metaplasia:aberrant expression of transcription factors and stem cell intestinalization. Gastric Cancer 2006; 9: 156 - 166

[6] Kobayashi M, Hashinoto S, Nishikura K et al. Magnifying narrow-band imaging of surface maturation in early differentiated-type gastric cancers after Helicobacter pylori eradication. J Gastroenterol 2013; 48: $1332-1342$

[7] Uedo $\mathrm{N}$, Ishihara $\mathrm{R}$, lishi $\mathrm{H}$ et al. A new method of diagnosing gastric intestinal metaplasia: narrow-band imaging with magnifying endoscopy. Endoscopy 2006; 38: 819-824
[8] Eda A, Osawa H, Yanaka I et al. Expression of homeobox gene CDX2 precedes that of $\mathrm{CDX} 1$ during the progression of intestinal metaplasia. J Gastroenterol 2002; 37: $94-100$

[9] Satoh K, Mutoh H, Eda A et al. Aberrant expression of CDX2 in the gastric mucosa with and without intestinal metaplasia: effect of eradication of Helicobacter pylori. Helicobacter 2002; 7: 192-198

[10] Hayakawa M, Nishikura K, Ajioka Y et al. Re-evaluation of phenotypic expression in differentiated-type early adenocarcinoma of the stomach. Pathol Int 2017; 67: $131-140$

[11] Mizoshita T, Inada K, Tsukamoto T et al. Expression of the intestinespecific transcription factors, $\mathrm{Cdx} 1$ and $\mathrm{Cdx} 2$, correlates shift to an intestinal phenotype in gastric cancer cells. J Cancer Res Clin Oncol 2004; 130: $29-36$

[12] Mizoshita T, Tsukamoto T, Nakanishi $\mathrm{H}$ et al. Expression of Cdx2 and the phenotype of advanced gastric cancers: relationship with prognosis. J Cancer Res Clin Oncol 2003; 129: 727 - 734

[13] Mizoshita T, Tsukamoto T, Inada K et al. Immunohistochemically detectable $\mathrm{Cd} \times 2$ is present in intestinal phenotypic elements in early gastric cancers of both differentiated and undifferentiated types, with no correlation to non-neoplastic surrounding mucosa. Pathol Int 2004; 54: $392-400$

[14] Park DY, Srivastava A, Kim GH et al. CDX2 expression in the intestinaltype gastric epithelial neoplasia: frequency and significance. Mod Pathol 2010; 23: 54-61

[15] Guo RJ, Suh ER, Lynch JP. The role of Cdx proteins in intestinal development and cancer. Cancer Biol Ther 2004; 3: 593-601

[16] Lauren P. The two histological main types of gastric carcinoma: Diffuse and so-called intestinal-type carcinoma. An attempt at a histoclinical classification. Acta Pathol Microbiol Scand 1965; 64: 31-49

[17] Nawata Y, Yagi K, Tanaka M et al. Reversal phenomenon on the mucosal borderline relates to development of gastric cancer after successful eradication of H. pylori. J Gastroenterol Hepatol Res 2017; 6: $1-6$

[18] Moribata K, Kato J, Iguchi M et al. Endoscopic features associated with development of metachronous gastric cancer in patients who underwent endoscopic resection followed by Helicobacter pylori eradication. Dig Endosc 2016; 28: $434-442$

[19] Rugge M, Genta RM. Staging and grading of chronic gastritis. Hum Pathol 2005; 36: $228-233$

[20] Rugge M, Correa P, Di Mario F et al. OLGA staging for gastritis: a tutorial. Dig Liver Dis 2008; 40: 650-658

[21] Capelle LG, de Vries AC, Haringsma J et al. The staging of gastritis with the OLGA system by using intestinal metaplasia as an accurate alternative for atrophic gastritis. Gastrointestinal Endosc 2010; 71: $11150-1158$

[22] Saka A, Yagi K, Nimura S. OLGA- and OLGIM-based staging of gastritis using narrow-band imaging magnifying endoscopy. Dig Endosc 2015; 27: $735-742$ 